\title{
DINÂMICA DA COMUNIDADE ARBÓREA EM UM FRAGMENTO DE FLORESTA ESTACIONAL SEMIDECIDUAL MONTANA EM LAVRAS, MINAS GERAIS, EM DIFERENTES CLASSES DE SOLOS ${ }^{1}$
}

Pedro Higuchi ${ }^{2}$, Ary Teixeira Oliveira-Filho ${ }^{3}$, Ana Carolina da Silva², Evandro Luiz Mendonça Machado ${ }^{4}$, Rubens Manoel dos Santos ${ }^{5}$, Daniel Salgado Pifano ${ }^{6}$

\begin{abstract}
RESUMO - Com o objetivo de verificar a existência de mudanças estruturais e a influência de diferentes classes de solos sobre as taxas de dinâmica da comunidade arbórea, um fragmento de Floresta Estacional Semidecidual Montana (8,7 ha) em Lavras, MG, foi estudado em um período de cinco anos (2000-2005). Os dados foram coletados em 47 parcelas de $20 \times 20 \mathrm{~m}$, dispostas em duas transeções, distantes $80 \mathrm{~m}$ entre si, cruzando o fragmento no sentido de maior comprimento. No ano de 2000, foram amostrados todos os indivíduos arbóreos com DAP $\geq 5 \mathrm{~cm}$. As informações coletadas para cada indivíduo foram: identificação botânica da espécie e DAP. Em 2005, foram registrados os indivíduos mortos, remensurados os sobreviventes e mensurados e identificados os indivíduos recrutados ( $\mathrm{DAP} \geq 5 \mathrm{~cm}$ ). Foram calculadas as taxas de dinâmica: mortalidade, recrutamento, ganho e perda em área basal de cada parcela, para a área total e para cada classe de solo (Nitossolos, Latossolos e Cambissolos). O padrão observado no fragmento foi de redução no número de indivíduos e estabilidade da área basal. Entretanto, não foram identificadas diferenças entre as classes de solos, em relação às taxas de dinâmica. As populações das espécies classificadas como de subdossel aumentaram a dominância ecológica na área. Os resultados permitiram concluir que o fragmento estudado está em uma fase avançada de sucessão pósdistúrbio e as variações espaciais das taxas de dinâmica não apresentaram relações com as classes de solos identificadas na área.
\end{abstract}

Palavras-chave: Dinâmica florestal, fatores edáficos e fragmento florestal.

\section{TREE COMMUNITY DYNAMICS IN A MONTANE SEMI-DECIDUOUS TROPICAL FOREST FRAGMENT IN LAVRAS, MINAS GERAIS, ON DIFFERENT SOIL CLASSES}

\begin{abstract}
In order to analyze the existence of structural changes and the influence of different soils classes on tree community dynamics rates, a fragment of tropical montane seasonal semi-deciduous forest $(8,7$ ha) in SE Brazil was studied in a 5 years period (2000-2005). Data were collected in 47, $20 \times 20 \mathrm{~m}$, plots arranged in two $80 \mathrm{~m}$ distant transect, crossing the study area toward its widest length. In 2000, were surveyed all individuals with $D B H \geq 5 \mathrm{~cm}$. Species botanical identification were provided and diameters at breast height $(D B H)$ were collected for each tree individual. In 2005, all dead trees were recorded, the surviving trees were remeasured and the recruits were measured and identified $(\mathrm{DBH} \geq 5 \mathrm{~cm})$. Mortality, recruitments, basal area loss and gain rates were calculated for each plot, for the total area and for each soil class (Nitosols, Oxysols and Cambisols). The overall pattern in the fragment indicated reduction in number of individuals
\end{abstract}

\footnotetext{
${ }^{1}$ Recebido em 29.04.2007 e aceito para publicação em 19.05.2008.

${ }^{2}$ Departamento de Fitotecnia da Universidade do Estado de Santa Catarina (UDESC). Lages-SC. E-mail: <phiguchi@ cav.udesc.br>.

${ }^{3}$ Departamento de Ciências Florestais da Universidade Federal de Lavras (UFLA). Lavras-MG. E-mail: <ary@ufla.br>.

${ }^{4}$ Pós-Doutorado do Departamento de Engenharia Florestal da Universidade de Brasília.

${ }^{5}$ Programa de Pós-Graduação em Engenharia Florestal da UFLA.

${ }^{5}$ Graduação em Ciências Biológicas da Universidade Federal de Juiz de Fora (UFJF), Juiz de Fora-MG.
} 
and basal area stability. However, there were no differences among soils classes regarding dynamics rates. The species populations classified as sub-canopy tended to increase its ongoing ecological dominance in the area. The results allowed us to conclude that the studied fragment is in an advanced successional phase post-disturbance and that spatial variations in dynamics rates had no relation with soils classes.

Keywords: Edaphic factors, forest dynamics and forest fragment.

\section{INTRODUÇÃO}

A Mata Atlântica, hoje com apenas $7 \%$ de sua área original (TABARELLI et al., 2005), é considerada um dos ecossistemas mais ameaçados no mundo, devido ao seu avançado estado de degradação e fragmentação. Mesmo assim, os fragmentos remanescentes são importantes por apresentarem elevada diversidade residual (TABARELLI et al., 1999), desempenharem serviços ambientais, como proteção do solo, e atuarem sobre o ciclo hidrológico, alterando os padrões de infiltração do solo e evapotranspiração (LINO e DIAS, 2003).

Considerando o cenário em que se encontra este importante bioma, estudos sobre dinâmica de comunidades arbóreas em fragmentos florestais são fundamentais, pois permitem o monitoramento e a previsão dos processos de transformação das populações e das comunidades vegetais isoladas. Do ponto de vista de aplicações práticas, esses estudos são importantes para fornecer informações que sirvam como subsídio para o manejo, visando à conservação desses remanescentes florestais (SHEIL et al., 2000).

Os estudos de dinâmica florestal geralmente são realizados por meio de dados provenientes de censos em parcelas permanentes, onde se realizam a contagem, medição, posterior recontagem e remedição de indivíduos sobreviventes (perdas e ganhos), sendo essas informações sumarizadas em taxas de mortalidade, recrutamento e rotatividade da comunidade (SHEIL e MAY, 1996). Vários trabalhos em florestas tropicais têm mostrado que os parâmetros observados, como as taxas de mortalidade e de recrutamento, apresentam variações espaciais (LIEBERMAN e LIEBERMAN, 1987) e temporais (MANOKORAN e KOCHUMMEN, 1987). Porém, as causas dessas variações são ainda desconhecidas, não estando claro quanto é de origem determinística ou estocástica (REZENDE, 2002), o que reforça a necessidade de melhor conhecer as relações entre as taxas de dinâmica de comunidades arbóreas e variáveis ambientais locais.
Em se tratando de florestas estacionais semideciduais, pouco se conhece sobre os impactos causados pela fragmentação sobre o componente arbóreo (PEREIRA et al., 2007; SILVAet al., 2004). No Estado de Minas Gerais, apesar da crescente consciência sobre a importância dos recursos florestais e sobre a urgência de conservá-los, são raros os trabalhos que investigam a dinâmica em ambientes fragmentados e sua interação com variáveis ambientais (OLIVEIRA-FILHO et al., 2007), o que reforça a necessidade de estudos nesses ambientes.

Dentre os fatores ambientais que influenciam a dinâmica de fragmentos florestais na Região Sudeste do Brasil, têm-se destacado o histórico de perturbação (GOMES et al., 2003), o efeito de borda (OLIVEIRAFILHO et al., 2007) e a topografia (APOLINÁRIO et al., 2005). Características do solo como fertilidade (BAKER et al., 2003) e saturação por água (JOLY, 1991) também exercem grande influência sobre os padrões de dinâmica observados em florestas.

Em geral, espécies arbóreas apresentam maiores taxas de crescimento em solos úmidos, de boa aeração e ricos em nutrientes (PEREIRA et al., 1999). Em fragmentos com alta disponibilidade de nutrientes no solo pode haver o favorecimento das espécies que apresentam elevada taxa de crescimento durante os estágios iniciais de sucessão, pois as espécies com baixas taxas de crescimento tendem a responder menos ao aumento nos níveis de nutrientes (DENSLOW et al., 1998).

Dessa forma, este estudo tem como objetivo avaliar a dinâmica da comunidade arbórea de um fragmento de Floresta Estacional Semidecidual Montana Secundária em Lavras, Minas Gerais, com a finalidade de fornecer subsídios para ações que visem ao manejo e à conservação de remanescentes. As hipóteses testadas foram: (i) em função do histórico de perturbação, a vegetação arbórea que constitui o fragmento se encontra em fase de mudança estrutural; e (ii) a dinâmica da comunidade arbórea apresenta variações espaciais, refletindo as diferentes classes de solos identificadas na área. 


\section{MATERIAL E MÉTODOS}

\subsection{Caracterização da área de estudo}

O estudo foi realizado em um Fragmento de Floresta Estacional Semidecidual Montana conhecido como "Mata da Subestação", situado no Campus da Universidade Federal de Lavras (UFLA). A "Mata da Subestação" está localizada no Município de Lavras, MG, nas coordenadas de $21^{\circ} 13^{\prime} 1729^{\prime \prime} \mathrm{S}$ e $44^{\circ} 57^{\prime} 47^{\prime \prime} \mathrm{W}$, com altitude variando entre 889,77 e $948,23 \mathrm{~m}$. O fragmento cobre uma área de aproximadamente 8,75 ha e reveste uma encosta de morro, apresentando, de acordo com Espírito-Santo et al. (2002), pedoforma do tipo convexo-convexa (Figura 1).
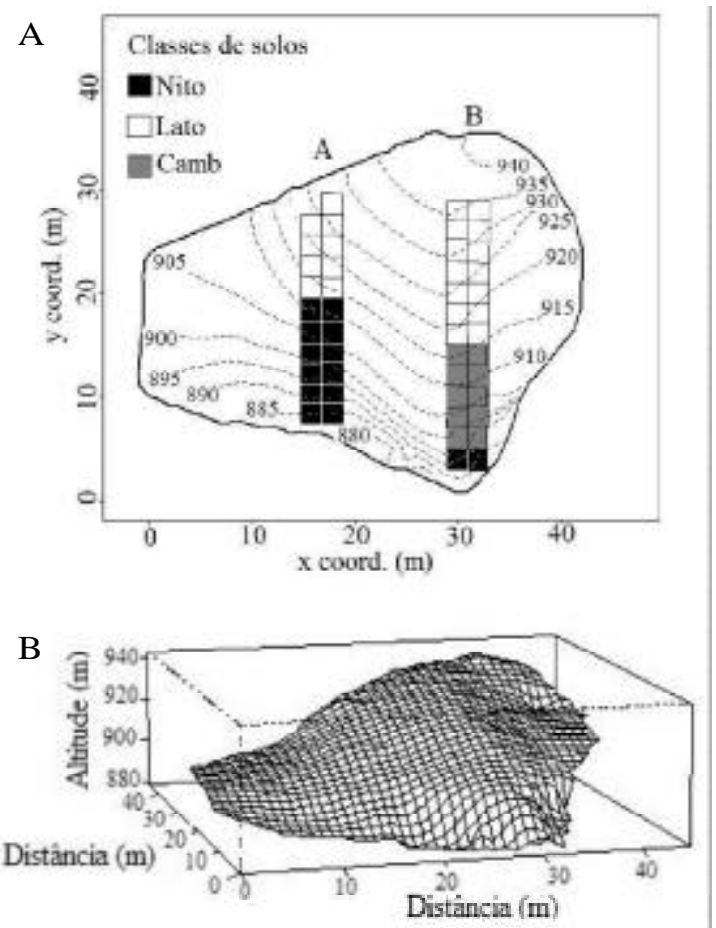

Figura 1 - Fragmento de floresta estacional semidecidual montana em Lavras, Minas Gerais: (A) Representação das duas transeções amostradas no interior do fragmento, com as classes de solos amostradas (Nito $=$ Nitossolos, Lato $=$ Latossolos e Camb $=$ Cambissolos) e (B) Diagrama de superfície mostrando o relevo do fragmento.

Figure 1-Montane seasonal semi-deciduous forest fragment in Lavras, MG, SE Brazil: (A) Representation of the two sampling transects in the fragment interior, with soil classes sampled; and (B) Surface diagram representing fragment topography.
$\mathrm{O}$ fragmento estudado, que se encontra inserido em uma matriz composta predominantemente por áreas agrícolas, tem um histórico comum a muitos outros fragmentos florestais localizados na região. Na década de 1950, motivados pela iminente desapropriação do local, os proprietários realizaram um corte raso na área. Desse então, o fragmento se regenerou de forma muito rápida, devido à brotação de cepas, e foi explorado esporadicamente para a retirada de lenha (ESPÍRITOSANTO et al., 2002).

O clima da região é do tipo Cwa, de Köppen, com precipitação e temperaturas médias anuais de 1.343,3 mm e $19,3^{\circ} \mathrm{C}$, respectivamente, com $76 \%$ da precipitação ocorrendo no período de novembro a fevereiro (BRASIL, 1992).

\subsection{Amostragem da vegetação}

Estudos sobre a florística e a estrutura da vegetação, assim com suas interações com fatores ambientais, foram realizados por Espírito-Santo et al. (2002), sendo a comunidade arbórea amostrada, no ano de 2000, em duas transeções de $40 \mathrm{~m}$ de largura, paralelas e distantes $80 \mathrm{~m}$ entre si, totalizando 2,8 ha de área amostrada. As transeções A e B foram subdivididas, respectivamente, em 21 e 31 parcelas contíguas de $20 \times 20 \mathrm{~m}$, de forma a representar a maior variação aparente do gradiente fisionômico da vegetação, ou seja, cruzando o fragmento no sentido de maior declividade da encosta e ligando bordas opostas (Figura 1). Em cada parcela foram registradas informações de todos os indivíduos vivos e com diâmetro à altura do peito (DAP), medido a $1,30 \mathrm{~m}$ de altura do solo, superior ou igual a $5 \mathrm{~cm}$. As informações registradas foram a identidade botânica das espécies e o DAP. Os materiais botânicos não reconhecidos no campo foram coletados e identificados no herbário da UFLA. A classificação botânica das espécies baseou-se no Angiosperm Phylogeny Group II (APG II, 2003).

No ano de 2005 foi realizado o segundo inventário da comunidade arbórea, utilizando-se a mesma metodologia do inventário anterior. Entretanto, em função da construção da Estrada do Contorno, que liga o Município de Ijaci à BR-265, e de constantes perturbações antrópicas, cinco parcelas localizadas na extremidade superior da transeção B foram eliminadas deste estudo. Foram incorporados os novos indivíduos que atingiram 
a classe de diâmetro estipulada (DAP $\geq 5 \mathrm{~cm}$ ), que foram identificados, medidos e individualizados com plaquetas. Os indivíduos mortos foram registrados e os sobreviventes, mensurados novamente.

\subsection{Características do solo}

A base de dados foi composta pela caracterização do ambiente edáfico conduzida por Espírito-Santo et al. (2002) na área em questão. Os solos foram classificados, segundo o Sistema Brasileiro de Classificação do Solo (EMBRAPA, 1999), como Nitossolos Vermelhos e Cambissolos Háplicos, tipos de solos presentes na baixa encosta, e Latossolos Vermelho-Amarelos, na alta encosta, sendo denominados, daqui para frente, apenas como Nitossolos (Nito), Cambissolos (Camb) e Latossolos (Lato), respectivamente.

\subsection{Dinâmica da comunidade arbórea}

Foram calculadas, para a amostra total e para as amostras definidas pelas classes de solos (Nitossolos, Latossolos e Cambissolos), as taxas de mortalidade e recrutamento e as taxas de ganho e perda em área basal, com base no número de indivíduos e área basal, por meio dos modelos algébricos (SHEIL e MAY, 1996):

$M=\left(1-\left(\left(\mathrm{N}_{0}-m\right) / N_{0}\right)^{1 / t}\right) \times 100$,

$R=\left(1-(1-r / N t)^{1 / \mathrm{t}}\right) \times 100$,

$P=\left(1-\left[\left(A B_{0}-\left(A B_{\mathrm{m}}+A B_{\mathrm{d}}\right)\right) / A B_{0}\right)^{1 / \mathrm{t}}\right) \times 100, \mathrm{e}$

$G=\left(1-\left[1-\left(A B_{\mathrm{r}}+A B_{\mathrm{g}}\right) / A B_{\mathrm{t}}\right)^{1 / \mathrm{t}}\right) \times 100$.

em que: $M=$ taxa de mortalidade anual; $R=$ taxa de recrutamento anual; $P=$ taxa de perda em área basal anual; $G=$ taxa de ganho em área basal anual; $t=$ intervalo de tempo entre inventários; $N_{\mathrm{o}}=$ número inicial de árvores; $N_{\mathrm{t}}=$ número final de árvores sobreviventes depois de $t ; m=$ número de árvores mortas; $r=$ número de árvores recrutadas; $A B_{\mathrm{o}}=$ área basal inicial; $A B_{\mathrm{t}}=$ área basal final depois de $t ; A B_{\mathrm{m}}$ = área basal das árvores mortas; $A B_{\mathrm{d}}=$ perda em área basal (redução diamétrica e perda parcial de troncos); $A B_{\mathrm{r}}=$ área basal de árvores recrutadas; e $A B_{\mathrm{g}}=$ ganho em área basal (crescimento das árvores).

Foram realizadas, em caráter exploratório, análises geoestatísticas com o objetivo de verificar a existência de dependência espacial entre as taxas de dinâmica.
Para isso, variáveis espaciais, correspondendo a coordenadas $\mathrm{x}$ e y do centro de cada parcela, foram utilizadas para definir uma rede de pontos. Essa rede permitiu a análise da co-variância espacial a partir de semivariogramas elaborados com base nos seguintes modelos estacionários isotrópicos: esférico, exponencial e gaussiano (CAMARGO, 1997).

As taxas de dinâmica da comunidade arbórea foram comparadas entre as diferentes classes de solos, por meio da análise de variância, ANOVA (ZAR, 1996). As diferenças entre as proporções de indivíduos mortos e recrutados na área total e na classes de solos foram comparadas pelo teste Binomial, um teste não-paramétrico que assume distribuição dicotômica (SOKAL e ROHLF, 1994).

A dinâmica das árvores por classe de diâmetro, nos setores amostrados, foi analisada empregandose intervalos de classe com amplitudes crescentes (5$10 \mathrm{~cm},>10-20 \mathrm{~cm},>20-40 \mathrm{~cm}$ e $>40-80 \mathrm{~cm}$ ), intervalos que têm sido adotados em estudos de dinâmica florestal na região(OLIVEIRA-FILHO et al., 2007) para compensar o acentuado declínio, em densidade, das classes de tamanhos maiores, típico da distribuição exponencial negativa (J-invertido). Em cada classe de diâmetro foram contabilizados o número de árvores mortas, o número de ingressantes (indivíduos recrutados e vindos de outras classes de diâmetro) e o número de egressantes (indivíduos que migraram para outras classes de diâmetro), podendo as duas últimas categorias ser progressivas ou regressivas (LIEBERMAN et al., 1985).

Com base na distribuição de diâmetros em 2000, foram utilizados testes G (SOKAL e ROHLF, 1994) para verificar se as freqüências de árvores vivas em 2005 e de árvores mortas foram independentes das classes de diâmetro. A diferença entre as proporções de árvores ingressantes (recrutas + imigrantes) e árvores egressas (mortos + emigrantes), em cada classe de diâmetro nos setores e na amostra total, foi verificada por meio do teste Binomial (SOKAL e ROHLF, 1994).

\subsection{Dinâmica das populações arbóreas}

Devido ao fato de a maior parte das espécies possuir poucos indivíduos por parcelas, foram escolhidas, para análise da dinâmica populacional na amostra total, as 12 populações mais abundantes em $2000(\mathrm{~N} \geq 75)$. A diferença entre a proporção dos números de recrutas e mortos, em cada uma das 12 
populações, foi verificada por meio do teste binomial (SOKAL e ROHLF, 1994).

Com o propósito de auxiliar a identificação de padrões ecologicamente significativos, as 12 espécies foram classificadas segundo sua preferência pelas classes de solos e sua guilda de regeneração. Os habitats preferenciais foram definidos com o auxílio do teste G (SOKAL e ROHLF, 1994) aplicado às abundâncias das espécies, em 2000, nas parcelas presentes nas diferentes classes de solos. As guildas de regeneração, adaptadas de Lieberman e Lieberman (1987), foram: (a) "sub-bosque", (b) "subdossel”, (c) "dossel-tolerante à sombra", (d) "dossel-exigente de luz" e (e) "pioneira". A classificação das 12 espécies nessas categorias se apoiou na experiência de campo dos autores e no trabalho de Espírito-Santo et al. (2002).

\section{RESULTADOS E DISCUSSÃO}

\subsection{Dinâmica da comunidade arbórea}

Durante o período de estudo foi observado na área total tendência de declínio no número de árvores e estabilidade em área basal. Essa tendência é reforçada pelo fato de a proporção de indivíduos mortos ter sido maior do que a de indivíduos recrutados para a área total, como demonstrado pelo teste Binomial $(p=0,0166)$ (Quadro 1). Quando analisada isoladamente, a proporção de recrutas e de árvores mortas não diferiu significativamente em relação às classes de solos ( $p$ $=0,1133, p=0,1950$ e $p=0,2267$, respectivamente para Cambissolos, Latossolos e Nitossolos) (Quadro 1). A estabilidade da área basal, apesar da redução em densidade na área total, ocorreu, principalmente, devido ao crescimento acentuado das árvores sobreviventes (Quadro 1).

Esse padrão caracterizado pela redução do número de indivíduos e manutenção ou aumento da área basal, devido ao crescimento das árvores sobreviventes, é típico de florestas tropicais em recuperação pós-distúrbio e em estádio avançado de sucessão (PHILLIPS et al., 2002). A mortalidade de indivíduos pode ter ocorrido devido ao processo de autodesbaste, em função da competição interespecífica entre as árvores que estão se desenvolvendo na área. Trabalhos realizados em fragmentos florestais no Sudeste do Brasil (APPOLINÁRIO et al., 2005; OLIVEIRA-FILHO et al., 2007) têm evidenciado que esse padrão é muito comum em fragmentos protegidos de distúrbios antrópicos. Entretanto, o padrão difere do observado em florestas maduras na Amazônia, definido pelo aumento na densidade de árvores e área basal (PHILLIPS et al., 2004). Enquanto a instabilidade neste estudo está claramente relacionada ao processo sucessional pósdistúrbio, uma vez que o fragmento sofreu corte raso na década de 1950 (ESPÍRITO-SANTO et al., 2002), na Amazônia, é sugerida como o resultado do aumento nas concentrações de $\mathrm{CO}_{2}$ na atmosfera (PHILLIPS e GENTRY, 1994), já que os estudos mencionados se concentraram em áreas prístinas.

Uma vez visto, a partir das análises exploratórias geoestatísticas, que as variáveis de dinâmica não apresentaram autocorrelação espacial, verificou-se, por meio de análise de variância (ANOVA), que, apesar de ser elemento importante sobre os padrões de estrutura do componente arbóreo e distribuição de espécies na área de estudo (ESPÍRITO-SANTO et al., 2002), as classes de solos não tiveram influência sobre as taxas de dinâmica (Quadro 1). A variação espacial das taxas de dinâmica está relacionada a outros fatores ambientais, como formação de clareiras naturais (ALVAREZ-BUYLLA, 1994), efeito de borda(OLIVEIRAFILHO et al., 2007) e distúrbios passados (OLIVEIRAFILHO et al., 1997).

Apesar de a mortalidade de indivíduos ter sido maior nas menores classes de diâmetro na área total $(\mathrm{G}=10,88 ; \mathrm{p}=0,01239)$ e em Latossolos $(\mathrm{G}=9,5191$; $p=0,02313$ ), o padrão de distribuição diamétrica dos indivíduos arbóreos não diferiu, entre 2000 e 2005, tanto na área total $(\mathrm{G}=0,1853 ; p=0,98)$, quanto nas classes de solos analisadas isoladamente $(\mathrm{Camb}-\mathrm{G}=0,048$; $p=0,1577 ;$ Lato $-\mathrm{G}=0,2818 ; p=0,9634 ;$ Nito $-\mathrm{G}=$ 0,$6339 ; p=0,2635$ ) (Quadro 2).

Esses resultados indicam que a redução em densidade de indivíduos, resultante do processo de autodesbastes, ocorreu, preferencialmente, nas classes de menor diâmetro, sobretudo nos Latossolos. Gomes et al. (2003) e Werneck e Franceschinelli (2004), estudando fragmentos florestais secundários com intenso histórico de perturbação na Região Sudeste, encontram o mesmo padrão de maior mortalidade nas menores classes diamétricas. Esse é mais um indicativo de que o fragmento está em processo de recuperação pós-distúrbio ambiental, uma vez que o esperado em florestas maduras é mortalidade independente da classe diamétrica (SWAINE et al., 1987).

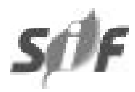

R. Árvore, Viçosa-MG, v., n.32, p.417-426, 2008 
Quadro 1 - Dinâmica da comunidade arbórea, no período de 2000-2005, em um fragmento de floresta semidecídual montana em Lavras, MG, para a amostra total e suas classes de solos

Table 1 - Tree community dynamics in a fragment of tropical montane seasonal semi-deciduous forest surveyed in 2000 2005 in Lavras, SE Brazil, for the total sample and its soil classes

\begin{tabular}{|c|c|c|c|c|c|c|}
\hline & Área Total & Cambissolos & Latossolos & Nitossolos & $F$ & $p(>F)$ \\
\hline \multicolumn{7}{|l|}{ Amostragem } \\
\hline Número de parcelas & 47 & 10 & 23 & 14 & & \\
\hline \multicolumn{7}{|l|}{ Número de Árvores } \\
\hline Inicial $2000\left(\mathrm{n}^{0}\right)$ & 2870 & 666 & 1374 & 830 & & \\
\hline Final $2005\left(n^{0}\right)$ & 2819 & 650 & 1355 & 814 & & \\
\hline Mudança líquida (\%) & $-1,8$ & $-2,4$ & $-1,4$ & $-1,9$ & 0,0660 & 0,9364 \\
\hline Sobreviventes $\left(\mathrm{n}^{\circ}\right)$ & 2626 & 613 & 1268 & 745 & & \\
\hline Mortas $\left(\mathrm{n}^{0}\right)$ & $244 *$ & 53 & 106 & 85 & & \\
\hline Recrutas $\left(\mathrm{n}^{\circ}\right)$ & 193 & 37 & 87 & 69 & & \\
\hline Tx. Mort . $\left(\%\right.$ ano $\left.^{-1}\right)$ & 1,8 & $1,6[1,6]$ & $1,6[1,6]$ & $2,1[2,1]$ & 0,7135 & 0,4955 \\
\hline Tx. Recr. $\left(\%\right.$ ano $\left.^{-1}\right)$ & 1,4 & $1,2[1,2]$ & $1,3[1,3]$ & $1,8[1,7]$ & 1,6155 & 0,2104 \\
\hline \multicolumn{7}{|l|}{ Área Basal } \\
\hline Inicial, $2000\left(\mathrm{~m}^{2}\right)$ & 53,700 & 12,197 & 25,506 & 15,997 & & \\
\hline Final, $2005\left(\mathrm{~m}^{2}\right)$ & 53,569 & 12,189 & 25,536 & 15,844 & & \\
\hline Mudança líquida (\%) & $-0,2$ & $-0,1$ & 0,1 & $-1,0$ & 0,0096 & 0,9904 \\
\hline $\operatorname{Mortas}\left(\mathrm{m}^{2}\right)$ & 3,559 & 0,926 & 1,425 & 1,207 & & \\
\hline Recrutas $\left(\mathrm{m}^{2}\right)$ & 0,542 & 0,103 & 0,241 & 0,198 & & \\
\hline Crescimento Sobrev. $\left(\mathrm{m}^{2}\right)$ & 2,886 & 0,815 & 1,214 & 0,857 & & \\
\hline Tx. de perdas $\left(\%\right.$ ano $\left.^{-1}\right)$ & 2,1 & $2,1[2,1]$ & $1,9[1,9]$ & $2,4[2,4]$ & 0,4401 & 0,6468 \\
\hline Tx. de ganho $\left(\%\right.$ ano $\left.^{-1}\right)$ & 2,1 & $2,1[2,1]$ & $1,9[1,9]$ & $2,3[2,3]$ & 2,1189 & 0,1323 \\
\hline
\end{tabular}

Tx. = taxa; Sobrev. = sobreviventes; $*$ = significância do teste binomial $(p=\langle 0,01$; símbolo no maior valor). Valores entre colchetes são as médias das $N$ parcelas de cada setor amostrado. $F$ e $p(>F)$ representam o nível de significância da análise de variância (ANOVA) entre os setores.

\subsection{Dinâmica das populações arbóreas}

Das 12 populações com maior abundância em 2000 , cinco (Calycorectes acutatus, $\mathrm{G}=0,88$ e $p$ $=0,646$, Mollinedia widgrenii, $\mathrm{G}=3,30, p=0,192$, Bauhinia longifolia, $\mathrm{G}=3,47, p=1,77$, Ixora warmingii, $\mathrm{G}=4,38, p=0,111$ e Myrcia splendens, $\mathrm{G}=2,98$ e $p=0,225$ ) foram indiferentes em relação ao tipo de solo, quatro foram preferenciais por Cambissolos (Galipia jasminifolia, $\mathrm{G}=149,09 \mathrm{e}$ $p<0,001$ Sebastiana commersoniana, $\mathrm{G}=49,32 \mathrm{e}$ $p<0,001$, Machaerium stipitatum, $\mathrm{G}=15,74 \mathrm{e} p<0,001$, e Platycyamus regnellii, $\mathrm{G}=9,12, p=0,010$ ), duas por Nitossolos (Casearia sylvestris, $\mathrm{G}=37,90 \mathrm{e}$ $p=<0,001$ e Cupania vernalis, $\mathrm{G}=20,73$ e $p=<0,001)$ e uma por Latossolos (Albizia polycephala, $\mathrm{G}=$ 8,20 e $p=0,017$ ) (Quadro 3).

Seis populações apresentaram diferenças significativas entre a proporção de indivíduos recrutados e mortos e três, maior recrutamento do que mortalidade, sendo todas classificadas como de subdossel: Galipia jasminifolia e Calycorectes acutatus e Ixora warmingii. As três espécies que tiveram maior proporção de indivíduos mortos do que recrutados foram classificadas como de dossel-exigente de luz: Albizia polycephala, Machaerium stipitatum e Myrcia splendens. A espécie que apresentou maior ganho em área basal foi Sebastiania commersoniana, classificada como de subdossel, e a de maior redução em área basal foi Myrcia splendens, considerada espécie de dossel-exigente de luz.

Os resultados indicaram que existiu tendência de as populações classificadas como de subdossel, como Galipia jasminifolia, aumentarem a dominância ecológica já existente na área, ao contrário dos fragmentos de Floresta Estacional Semidecidual estudados em Minas Gerais por Oliveira-Filho et al. (1997) e Paula et al. (2002). Esses autores observaram a tendência de que a dominância ecológica de populações 
Quadro 2 - Número de árvores, de mortos, de emigrantes (Emig.), de recrutas (Recr.) e de imigrantes (Imig.) por classe de diâmetro (DAP), entre 2000 e 2005, em um fragmento de floresta semidecídual montana em Lavras, MG, para a amostra total e classes de solos

Table 2 - Number of trees, dead trees, outgrowth (Emig.), and recruits (Recr.) per diameter (DAP = dbt) classes between 2000 and 2005 in a fragment of tropical montane seasonal semi-deciduous forest in Lavras, SE Brazil, for the total sample and soils classes

\begin{tabular}{|c|c|c|c|c|c|c|c|c|c|}
\hline \multirow{2}{*}{$\begin{array}{l}D A P \\
(\mathrm{~cm}) \\
\end{array}$} & \multicolumn{3}{|c|}{ Número de Árvores } & \multicolumn{2}{|c|}{ Mortos } & \multirow{2}{*}{$\frac{\text { Emig. }}{n^{o}}$} & \multirow{2}{*}{$\frac{\text { Recr. }}{n^{o}}$} & \multirow{2}{*}{$\frac{\text { Imig. }}{n^{o}}$} & \multirow{2}{*}{$\frac{\text { Teste Binomial }}{p}$} \\
\hline & 2000 & 2005 & Esp. & $n^{o}$ & Esp. & & & & \\
\hline \multicolumn{10}{|l|}{ Cambissolos } \\
\hline $5-10$ & 336 & 329 & $(327,9)$ & 33 & $(26,7)$ & 6 & 37 & 17 & 0,53 \\
\hline$>10-20$ & 239 & 233 & $(233,3)$ & 15 & $(19,0)$ & 18 & 0 & 9 & 0,44 \\
\hline$>20-40$ & 79 & 77 & $(77,1)$ & 3 & $(6,3)$ & 4 & 0 & 3 & 0.75 \\
\hline$>40-80$ & 12 & 11 & $(11,7)$ & 2 & $(0,9)$ & 1 & 0 & 0 & 0,33 \\
\hline Totais & 666 & 650 & & 53 & & 29 & 37 & 29 & \\
\hline \multicolumn{10}{|l|}{ Latossolos } \\
\hline $5-10$ & 706 & 691 & $(696,2)$ & 62 & $(54,5)$ & 9 & 87 & 49 & 0,33 \\
\hline$>10-20$ & 472 & 464 & $(465,4)$ & 38 & $(36,4)$ & 54 & 0 & 24 & 0,52 \\
\hline$>20-40$ & 168 & 171 & $(165,7)$ & 4 & $(13,0)$ & 16 & 0 & 9 & 0,71 \\
\hline$>40-80$ & 28 & 29 & $(27,6)$ & 2 & $(2,16)$ & 3 & 0 & 0 & 1,0 \\
\hline Totais & 1374 & 1355 & & 106 & & 82 & 87 & 82 & \\
\hline \multicolumn{10}{|l|}{ Nitossolos } \\
\hline $5-10$ & 417 & 402 & $(409,0)$ & 45 & $(42,7)$ & 8 & 69 & 47 & 0,28 \\
\hline$>10-20$ & 281 & 286 & $(275,6)$ & 31 & $(28,8)$ & 56 & 0 & 20 & 0,70 \\
\hline$>20-40$ & 118 & 112 & $(115,7)$ & 9 & $(12,1)$ & 15 & 0 & 12 & 0,41 \\
\hline$>40-80$ & 14 & 14 & $(13,7)$ & 0 & $(1,4)$ & 4 & 0 & 4 & 1,0 \\
\hline Totais & 830 & 814 & & 85 & & 83 & 69 & 83 & \\
\hline \multicolumn{10}{|l|}{ Área total: } \\
\hline $5-10$ & 1459 & 1422 & $(1433,1)$ & 140 & $(124,0)$ & 23 & 193 & 113 & 0,10 \\
\hline$>10-20$ & 992 & 983 & $(974,4)$ & 84 & $(84,3)$ & 128 & 0 & 53 & 0,62 \\
\hline$>20-40$ & 365 & 360 & $(358,5)$ & 16 & $(31,03)$ & 35 & 0 & 24 & 0.64 \\
\hline$>40-80$ & 54 & 54 & $(53,0)$ & 4 & $(4,6)$ & 8 & 0 & 4 & 1,0 \\
\hline Totais & 2870 & 2819 & & 244 & & 194 & 193 & 194 & \\
\hline
\end{tabular}

Esp = freqüências esperadas do número de árvores em 2005 e do número de mortos por classe de DAP com base na distribuição do número de árvores por classe de DAP, em 2000; $p=$ significância do teste binomial das proporções de saídas (mortos + emigrantes) e entradas (recrutas + imigrantes).

tolerantes à sombra sucedesse a dominância de populações exigentes de luz e pioneiras, ao longo do tempo.

Os resultados permitiram a conclusão de que o fragmento estudado se encontra em recuperação pósdistúrbio e em avançado estádio de sucessão, caracterizado pelos seguintes eventos: redução no número de indivíduos e estabilidade da área basal, devido ao crescimento das árvores sobreviventes, à mortalidade proporcionalmente maior nas classes de menores tamanhos e ao aumento da dominância ecológica já existente de populações tolerantes à sombra, o que confirma a primeira hipótese de que o fragmento está em fase de mudança estrutural.

Apesar de a mortalidade de indivíduos ter sido dependente da classe diamétrica nos Latossolos, sendo maior nas classes de menor diâmetro, a segunda hipótese testada, a de que as taxas de dinâmica da comunidade arbórea apresentam variações de acordo com as classes de solos, foi refutada. 
Quadro 3 - Número de indivíduos e área basal das 12 espécies mais abundantes em 2000, em um fragmento de floresta semidecidual montana em Lavras, MG

Table 3 - Number of trees and basal area of 12 tree species most abundant in 2000 in a fragment of tropical montane seasonal semi-deciduous forest in Lavras, SE Brazil

\begin{tabular}{|c|c|c|c|c|c|c|c|c|c|}
\hline \multirow[b]{2}{*}{ Espécies } & \multirow{2}{*}{$\frac{\text { Habitat }}{\text { preferencial }}$} & \multirow{2}{*}{$\frac{\text { Guilda de }}{\text { Regeneração }}$} & \multicolumn{4}{|c|}{ Número de Árvores } & \multirow{2}{*}{$\begin{array}{c}\text { Teste } \\
\text { Binomial }\end{array}$} & \multicolumn{2}{|c|}{$\begin{array}{c}\text { Área Basal } \\
\left(\mathrm{m}^{2}\right)\end{array}$} \\
\hline & & & $\mathrm{N} 1$ & $\mathrm{~N} 2$ & $\mathrm{~m}$ & $\mathrm{r}$ & & $\mathrm{AB} 1$ & $\mathrm{AB} 2$ \\
\hline Galipia jasminiflora (A.St.-Hil.) Engler & Camb & SubDos & 212 & 245 & 8 & 41 & $* * *$ & 1,079 & 1,256 \\
\hline Sebastiania commersoniana (Baill.) Smith \& Downs & Camb & SubDos & 157 & 154 & 12 & 9 & $\mathrm{~ns}$ & 1,228 & 1,778 \\
\hline Albizia polycephala (Benth.) Killip & Lato & DosLuz & 136 & 119 & 19 & 2 & $* * *$ & 1,998 & 1,961 \\
\hline Machaerium stipitatum Tul. & Camb & DosLuz & 128 & 117 & 15 & 4 & $* *$ & 1,385 & 1,389 \\
\hline Calycorectes acutatus (Miq.) Toledo & Indif & SubDos & 113 & 124 & 1 & 12 & $* * *$ & 1,089 & 1,264 \\
\hline Casearia sylvestris Swartz & Nito & Pio & 107 & 104 & 4 & 1 & $\mathrm{~ns}$ & 1,022 & 1,089 \\
\hline Cupania vernalis Cambess. & Nito & DosLuz & 87 & 86 & 5 & 4 & $\mathrm{~ns}$ & 0,932 & 1,001 \\
\hline Platycyamus regnellii Benth. & Camb & DosLuz & 85 & 82 & 4 & 1 & $\mathrm{~ns}$ & 1,602 & 1,826 \\
\hline Mollinedia widgrenii A. DC. & Indif & SubDos & 84 & 87 & 2 & 5 & $\mathrm{~ns}$ & 0,902 & 0,962 \\
\hline Bauhinia longifólia (Bongard) D.Dietrich & Indif & SubDos & 78 & 75 & 6 & 3 & $\mathrm{~ns}$ & 0,719 & 0,622 \\
\hline Ixora warmingii Müll.Arg & Indif & SubDos & 76 & 82 & 1 & 7 & $*$ & 1,030 & 1,217 \\
\hline Myrcia splendens (Swartz) DC. & Indif & DosLuz & 75 & 62 & 22 & 9 & $* *$ & 0,922 & 0,763 \\
\hline
\end{tabular}

Guildas regeneração: DosLuz = dossel-exigente de luz, SubDos = subdossel e Pio = pioneira. Número de árvores: N1 = inicial $(2000)$, $\mathrm{N} 2$ = final $(2005), \mathrm{m}=$ mortos e $\mathrm{r}=$ recrutas. Comparações por meio do teste binomial de m e $\mathrm{r}$ : a significância do teste $(p)$ é fornecida onde é pertinente $(* \mathrm{p}<0,10 ; * * \mathrm{p}<0,05 ; * * * \mathrm{p}<0,005 ; \mathrm{ns}=$ não-significativo $)$. Área basal: AB $1=\mathrm{inicial}(2000)$ e AB2 = final $(2005)$.

\section{AGRADECIMENTOS}

À CAPES, pela concessão da Bolsa de Doutorado ao primeiro autor e aos revisores anônimos, pela leitura crítica do manuscrito.

\section{REFERÊNCIAS}

ALVAREZ-BUYLLA, E. R. Density dependence and patch dynamics in tropical in tropical rain forest: matrix models and applications to a tree species. American Naturalist, v.143, n.1, p.155-191, 1994.

ANGIOSPERM PHYLOGENY GROUP II. An up date of the Angiosperm Phylogeny Group classification for the orders and families of flowering plants: APG II. Botanical Journal of Linnaean Society, v.141, p.399-436, 2003.

APPOLINÁRIO, V.; OLIVEIRA-FILHO, A. T.; GUILHERME, F. A. G. Tree population and community dynamics in a Brazilian tropical semideciduous forest. Revista Brasileira de Botânica, v.28, n.2, p.347-360, 2005.

BAKER, T. R.; BURSLEM, D. F. R. P.; SWAINE, M. D. Associations between tree growth, soil fertility and water availability at local and regional scale in Ghanaian tropical rain forest. Journal of Tropical Ecology, v.19, n.2, p.109-125, 2003.
BRASIL. Ministério da Agricultura e Reforma Agrária. Normais climatológicas 19611990. Brasília: 1992.

CAMARGO, E. C. G. Desenvolvimento, implementação e teste de procedimentos geoestatísticos (Krigeagem) no sistema de processamento de informações georeferenciadas (SPRING). 1997. 124f. Dissertação (Mestrado em Sensoriamento Remoto) Instituto Nacional de Pesquisa Espaciais, São José dos Campos, 1997.

DENSLOW, J. S.; ELLISON, A. M.; SANFORD, R. E. Treefall gap size effects on above and belowground process in a tropical wet forest. Journal of Ecology, v.86, p.597-609, 1998.

EMPRESA BRASILEIRA DE PESQUISA AGROPECUÁRIA - EMBRAPA. Centro Nacional de Pesquisa de Solo. Sistema brasileiro de classificação de solos. Rio de Janeiro: 1999.

ESPÍRITO-SANTO,F.D.B.; OLIVEIRA-FILHO, A.T.; MACHADO, E. L. M. Variáveis ambientais e a distribuição de espécies arbóreas em um remanescente de Floresta Estacional Semidecidual Montana no campus da Universidade Federal de Lavras, MG.Acta Botânica Brasileira, v.16, n.3, p.331-336, 2002. 
GOMES, E. P. C.; MANTOVANI, W.;

KAGEYAMA, P. Y. Mortality and recruitment of trees in a secondary montane rain forest in southeastern Brazil. Brazilian Journal of Biology, v.63, n.1, p.47-60, 2003.

JOLY, C. A. Flooding tolerance in tropical trees. In: HOLM-NIELSEN, L. B.; NIELSEN, I. C.; BALSLEV, H. (Eds.). Tropical forests: Botanical dynamics, speciation and diversity. London: Academic Press, 1991.

LIEBERMAN, D.; LIEBERMAN, M. Forest tree growth and dynamics at La Selva, Costa Rica (1969-1982). Journal of Tropical Ecology, v.3, NUMERO, 1987. p.347-358.

LIEBERMAN, D. et al. Mortality patterns and stand turnover rates in a wet tropical forest in Costa Rica. Journal of Ecology, v.73, n.3, p.915-924, 1985.

LINO, C. A.; DIAS. H. Águas e floresta da Mata Atlântica: por uma gestão integrada. São Paulo: Conselho Nacional da Reserva da Biosfera da Mata Atlântica e Fundação S. O. S Mata Atlântica, 2003.

MANOKARAN, N.; KOCHUMMEN, K. M. Recruitment, growth and mortality of the species in lowland dipterocarp forest in Peninsular Malaysia. Journal of Tropical Ecology, v.3, n.4, p.315-330, 1987.

OLIVEIRA-FILHO, A. T. et al. Dinâmica da comunidade e populações arbóreas da borda e interior de um remanescente florestal na Serra da Mantiqueira, Minas Gerais, em um intervalo de cinco anos (1994-2004). Revista Brasileira de Botânica, v.30, p.149-161, 2007.

OLIVEIRA-FILHO, A. T.; MELLO, J.M.; SCOLFORO, J. R. Effects of past disturbance and edges on tree community structure and dynamics within a fragment of tropical semideciduous forest in south-eastern Brazil over a five years period (1987-1992).Plant Ecology, v.131, n.1, p.45-66, 1997.

PAULA, A. et al. Alterações florísticas ocorridas num período de quatorze anos na vegetação arbórea de uma Floresta Estacional Semidecidual em Viçosa-MG. Revista Árvore, v.26, n.6, p.743-749, 2002.
PEREIRA, J. A. A.; ALVARENGA, S. A.; DAVIDE, A. C. Desenvolvimento de espécies florestais de rápido crescimento em diferentes sítios visando a recomposição de matas ciliares. Cerne, v.5, n.1, p.36-51, 1999.

PEREIRA, J. A. A.; OLIVEIRA-FILHO, A. T.; LEMOS-FILHO, J. P. Environmental heterogeneity and disturbance by humans control much of the tree species diversity of Atlantic montane forest fragments in SE Brazil. Biodiversity and Conservation, v.16, n.6, p.1761-1784, 2007.

PHILLIPS, O. L. et al. Pattern and process in Amazonian tree turnover, 1976 - 2001.

Philosofical Transactions of the Royal Society for London Serie B, v.359, n.1443, p.381-407, 2004.

PHILLIPS, O. L.; GENTRY, A. H. Increasing turnover through time in tropical forests. Science, v.263, n.5149, p.954-955, 1994.

PHILLIPS, O. L. et al. Changes in growth of tropical forests: Evaluating potential biases. Ecological Applications, v.12, n.2, p.576-587, 2002.

REZENDE, A. V. Diversidade, estrutura, dinâmica, prognose do crescimento de um cerrado sensu stricto submetido a diferentes distúrbios por desmatamento. 2002. 243f. Tese (Doutorado em Engenharia Florestal) - Universidade Federal do Paraná, Curitiba, 2002.

SHEIL, D.; JENNINGS, S.; SAVILL, P. Long-term permanent plot observations of vegetation dynamics in Bundongo, a Ugandan rain forest. Journal of Tropical Ecology, v.16, n.6, p.675-800, 2000.

SHEIL, D.; MAY, R. M. Mortality and recruitment rate evaluations in heterogeneous tropical forests. Journal of Ecology, v.84, n.1, p.91-100, 1996.

SILVA, C. T. et al. Avaliação temporal da florística arbórea de uma floresta secundária no Município de Viçosa, Minas Gerais. Revista Árvore, v.28, n.3, p.429-441, 2004. 
SOKAL, R. R.; ROHLF, F. J. Biometry: The principles and practice of statistics in biological research. 3.ed. New York: WH Freeman Company, 1994.

SWAINE, M. D.; LIEBERMAN, D.; PUTZ, F. E. The dynamics of tree populations in tropical forest: A review. Journal of Tropical Ecology, v.3, n.4, p.359-366, 1987.

TABARELLI, M.; MANTOVANI, W.; PERES, C. A. Effects of habitat fragmentation on plant guild structure in the montane Atlantic forest of southeastern Brazil. Biological

Conservation, v.91, n.2/3, p.119-127, 1999.
TABARELLI, M. et al. Challenges and opportunities for biodiversity conservation in the Brazilian Atlantic forest. Conservation Biology, v. 19, n.2/3, p.695-700, 2005.

WERNECK, M. S.; FRANCESCHINELLI, E. V. Dynamics of a dry forest fragment after exclusion of human disturbance in southeastern Brazil. Plant Ecology, v.174, p.337-346, 2004.

ZAR, J. H. Biostatistical analysis. 3.ed. New Jersey: Prentice-Hall, 1996. 